SHAHRIAR AFANDIZADEH ZARGARI, Ph.D. ${ }^{1}$

E-mail: zargari@iust.ac.ir

HAMID MIRZAHOSSEIN, Ph.D. Candidate ${ }^{1}$

E-mail: mirzahossein@iust.ac.ir

(Corresponding Author)

YI-CHANG CHIU, Ph.D. ${ }^{2}$

E-mail: chiu@email.arizona.edu

1 Department of Transportation Planning and Engineering,

School of Civil Engineering, Iran University of Science and

Technology Narmak, Tehran, 1684613114, Iran

2 Department of Civil Engineering and Engineering

Mechanics, College of Engineering, University of Arizona

1209 E. Second St., Tucson, Arizona 85721, USA
Traffic Planning

Preliminary Communication

Submitted: Dec. 7, 2015

Accepted: July 14, 2016

\title{
QUICK LINK SELECTION METHOD BY USING PRICING STRATEGY BASED ON USER EQUILIBRIUM FOR IMPLEMENTING AN EFFECTIVE URBAN TRAVEL DEMAND MANAGEMENT
}

\begin{abstract}
This paper presents a two-stage model of optimization as a quick method to choose the best potential links for implementing urban travel demand management (UTDM) strategy like road pricing. The model is optimized by minimizing the hidden cost of congestion based on user equilibrium ( $\mathrm{MH}$ CCUE). It forecasts the exact amount of flows and tolls for links in user equilibrium condition to determine the hidden cost for each link to optimize the link selection based on the network congestion priority. The results show that not only the amount of total cost is decreased, but also the number of selected links for pricing is reduced as compared with the previous toll minimization methods. Moreover, as this model just uses the traffic assignment data for calculation, it could be considered as a quick and optimum solution for choosing the potential links.
\end{abstract}

\section{KEY WORDS}

congestion; minimization; hidden cost; user equilibrium; link selection method; urban travel demand management (UTDM);

\section{INTRODUCTION}

Due to population growth in cities and the health care arguments, demand and willingness to use private vehicles (PV) has increased. Since the network expansion could not support all the PV demands and moreover, based on the Braess's paradox [1] it is not the best solution most of the time, the demand has been greater than the supply and this issue makes disequilibrium in urban transportation network. The result of this situation is the congestion and obstruction of network that causes various mental and environmental issues.
Preventing the congestion in urban transportation networks is one of the main concerns of transportation planners and traffic managers around the world. In response to this matter, they try to detail a stable condition and status for urban traffic network in microscopic and macroscopic scales. However, some studies improve the microscopic aspect of this issue by changing and refining traffic light schedules during congestion periods using internal/external traffic metering strategy [2], these kinds of strategies prove to be useful in the short term or for specific location of a sub-network under specific conditions. The main cause of congestion is lack of balance and an unsteady relationship between the supply and demand in the transportation sector. Thus, as previously discussed, increasing the supply alone will not provide a long-term solution, and most experts suggest using urban travel demand management (UTDM) strategies as a sustainable solution to control the demand for utilizing the transportation infrastructure more efficiently. In other words, UTDM strategies do not encourage decision makers to expand and widen the roads and highways as a solution for managing the traffic congestion that causes urban sprawl. Rather, they encourage people to use more efficiently the existing infrastructure.

Also, today sustainability is a controversial issue that affects social, environmental and economic aspects of human life. In this regard, since travel demand management (TDM) has been introduced as one of the solutions to reach sustainability [3], finding the reasonable location to implement its strategies and policies in urban transportation network would be the main or at least the first question for which the planners should have an answer. 
This research introduces an optimization problem to choose the potential roads - network links - that could be considered for TDM implementation based on congestion toll pricing theories by minimizing the hidden cost of congestion based on the user equilibrium (MHCUE) model. The concept of the hidden cost comes from congestion pricing principle. It is defined as the difference between the marginal social cost (the total cost that all the $n+1$ drivers experience based on the one further decision to travel including all private costs and also the cost experienced by people other than the driver who are exposed to the congestion and air pollution resulting from the car use) and the private cost (the cost experienced by the driver of the car, including the fuel and oil, maintenance and so on). Thus, the model by congestion toll pricing that is considered as one of the TDM methods, not only finds the potential links that could be considered a candidate for implementing the TDM, but it also investigates the amount of tolls that yield uncongested conditions for the network.

\section{BRIEF LITERATURE OVERVIEW}

Today's applications of TDM strategies are not only limited to facilitating shifts in travel time or travel mode - they also address shifts in travel routes [4]. Many studies show how transportation planners and traffic engineers try to implement TDM strategies in urban transportation networks [3, 5-9]. But, finding the location for implementing these strategies is one of the main concerns of planners when designing the selected policies. However, some policies, like congestion toll pricing as the first best congestion pricing where total costs in the city are minimized [10], could be instrumental in solving this issue if implemented properly. Congestion toll pricing is one of the most effective methods among TDM policies in urban transportation networks, which is to overcome two basic problems: congestion and environmental pollution.

Theory and practice have shown that receiving tolls, when designed and determined properly, can play a great role in reducing traffic congestion on links of urban transportation networks [11]. In other words, imposing tolls can help operators to manage the demand of travel and decrease urban traffic congestion and its negative effects such as total lost time of human resources, environmental pollutants, and so on.

The basic principle used in congestion toll pricing methods are based on notifying the users of the additional cost (marginal or hidden cost) they impose on other users [12]. In other words, congestion pricing recommends charges that force users to adopt these externalities to some extent, thus reducing or eliminating the deadweight loss [13]. In this case, the tolls are determined in a way that results in the possibility or impossibility of trip generation, shifting the trip from the peak to off-peak hours, or choosing alternate routes by users. Without considering the congestion toll pricing, users are not aware of the costs that they imposed on others by using the route, so by collecting tolls, in fact, users pay for the negative effects of their presence in the congested areas.

Determining congestion toll pricing on traffic networks is an old problem, which economists and transportation planners have studied extensively in the past years, and these studies have always had two main objectives. First, network pricing for determining the appropriate tolls to reduce or avoid the existing congestion, and second, funding for implementing transportation projects and their maintenance.

Toll collection experts, using certain assumptions, have implemented different methods to provide models for traffic demand management in networks in order to apply the pricing policies as well as possible. Pigou [14] was one of the pioneers who offered the tax model, and proposed the issue of marginal social cost pricing, and after him, Knight [15] expanded it for traffic models.

Although Knight provided the drivers route choice behaviour, it was Wardrop [16] who formulated the problems related to equilibrium using two principles, which are known as Wardrop principles. Later, Beckmann et al. [17] analysed equilibrium mathematical models in terms of formulas.

In static traffic assignment models, the demand between each pair of origin-destination are the constants and the constraints are system-balancing limitations, even though the travel rate between pairs of origin-destination is determined based on the function of minimum cost between each origin-destination. This is known as the traffic assignment problem with elastic demand [18], which means users have many choices, and they actually decide on the basis of their economic situation. In this case, the marginal cost toll is imposed on the users to change the flow pattern from user equilibrium to system optimum (SO) with fix demand. Thus, by assessing a proper toll on each user using a specific network link, the traffic flow situation will be close to system optimum in terms of minimizing the total travel cost. This solution could be appropriate for forecasting the potential links for implementing TDM strategies. Since, this approach tries to close the gap between UE to SO, its purpose is to eradicate congestion. Moreover, pricing has a big advantage over other TDM policies in that it encourages users and systems to adjust all aspects of their behaviour: number of trips, destination, mode of transport, time of day, route, and so on [19].

As the theoretical background of congestion toll pricing is based on the fundamental economic principles, this subject has had a lot of interest in recent years for both economists and transportation planners. Yang and Huang found a theoretical method to 
show how this classical economic principle could work in a congested urban network [20].

In this regard, different mathematical models for pricing are presented in research literature [21-25]; they propose a framework for congestion toll pricing with different objectives to calculate the marginal cost of congestion in an effective way, in which objectives are eventuated by some constraints that are imposed on the objective function. Some of the most famous ones are MinSys, MinMax, and MinTB models [21-23, 25]. The models are compared with the marginal social cost pricing (MSCP) that is the main traditional approach for congestion pricing. The models have been presented as a two stage optimization problem that all use the arc-node formulation of feasible flows.

For instance, the MinSys algorithm [21] aims to minimize the total tolls collected while constraining the toll vector to be non-negative and it contains two sections as a bi-level minimization problem. In step 1 , the model solves the system optimum problem to obtain an optimal solution represented as $v$ * by following equations:

$\min s(v)^{T} v$

Subject to:

$v=Z x$

$A x=b$

$x \geq 0$

In step 2 , the algorithm goes to minimize $\beta^{T} V^{*}$ over the polyhedron $w\left(v^{*}\right)$ that is defined by the following inequalities:

$$
\begin{aligned}
& Z^{T}\left(s\left(v^{*}\right)+\beta \geq A^{T} \rho\right. \\
& \left(v^{*}\right)^{T}\left(s\left(v^{*}\right)+\beta\right)=b^{T} \rho \\
& \beta \geq 0
\end{aligned}
$$

In these formulation parameters defined on the basis of the following notation, $g=(N, A)$ is a network with $N$ being the node and $A$ being the arc set; $\widehat{A}$ is the incidence matrix of $g ; b(k)$ is demand vector associated to each $k$ (commodity); $\beta$ is the toll vector; $x(k)$ is the commodity ( $k$ ) flow (variable) vector and the sum of all the commodity flow vectors becomes the aggregate flow vector denoted as $v$ and the cost of that aggregate flow is $s(v)$. Also, the system feasible flows are introduced as follows:

$$
\begin{aligned}
& v=\sum_{k} x(k) \\
& A x(k)=b(k) \quad \forall k \in K \\
& x(k) \geq 0 \quad \forall k
\end{aligned}
$$

In addition, in regard to the presented formulation for MinSys, other formulations of MinMax, and MinTB are presented in Hearn and Ramana research as the base study for comparing these formulations [22]. MinMax minimizes the maximum toll on the transportation network:

$\min z$

Subject to:

$$
\begin{aligned}
& (\beta, \rho) \in W\left(v^{*}\right) \\
& z \geq \beta_{a} \quad \forall a \in A \\
& \beta \geq 0
\end{aligned}
$$

Also, the MinTB minimizes the number of toll booths [25]. The formulation requires a positive constant $M$ that exceeds the largest toll and a vector $y$ of binary variable $y_{a}$. Its formulation is as follow:

$\min \sum_{a \in A} y_{a}$

Subject to:

$(\beta, p) \in W\left(v^{*}\right)$

$\beta_{a} \leq M z_{a} \quad \forall a \in A$

$y_{a} \in\{0,1\}$

$\beta \geq 0$

Although the traffic assignment problem that the previous models used refers to different optimization and equilibrium problems, in this paper we just mention the system optimum (SO) and the user equilibrium (UE). The readers are referred to [26] for more background about these kinds of traffic assignment problems.

In summary, most of the research on congested networks tries to evaluate the marginal cost or the hidden cost of using the urban transportation network by users. Finding the hidden cost is a very important issue for transport planners to manage the network by some TDM methods to encourage or discourage the people to use the roads more efficiently by considering the equilibrium condition that could be reached by congestion toll pricing. However, people want to pay for something that they want to earn and not for something that they do not want, like congestion [27], so finding the best price and solution for users based on the appropriate locations is absolutely essential. Moreover, people do not want to pay for something that they once used as a free service [28]. However, despite all the efforts in the last years, there is no general and optimized result for considering the congestion pricing 
models as the one solution to determine the potential TDM location in urban transportation network.

\section{PROBLEM SOLUTION}

The concept of minimizing the hidden cost of congestion based on the user equilibrium (MHCCUE) model is presented in this chapter. In this regard, the tolls in the whole network should be optimized based on the user equilibrium to find the most considerable links for implementing TDM policies on them.

As we know from the equilibrium concepts, and especially the expression of equilibrium in transportation, the user equilibrium is not the same as the optimized system situation and the total cost of the network in user equilibrium condition is more than the total cost of the network in optimized system situation. Therefore, the objective function in this model tries to optimize the flow based on the user equilibrium condition by solving two stages of optimization programming that in the first stage solve the traffic assignment to calculate the traffic flow on each link and then calculate the amount of dedicated toll for each link. In this model, as in the mentioned models in literature review, the hidden cost is imposed on the users according to value of time (VOT) - the value of one unit of time for users who pass the determined link - has an equal weight as travel time, for instance, and it can be one dollar per unit of time. As the previous models use the same value for time and imposed cost, this assumption is considered conservative and a better comparison between the results of proposed model and the ones introduced in the previous section.

\subsection{Objective function}

This section presents the model that has been developed in this research for quick estimation of the potential links that can be considered for TDM implementation. The model is a two-stage optimization problem that first solves the UE as a sub problem to find the link flows based on the user perspective and then solve the upper level to find the optimized hidden cost of congestion. Therefore, by finding the VOT that could be considered as a cost for the entire network, the model introduces the rational priority for links to be selected as a potential candidate for implementing the TDM policies in urban transportation network. The objective function and constraints are presented in Equations 20 to 27.

First:

Minimize $Z_{1}=\sum_{a \in A} \int_{0}^{x_{a}} t_{a}\left(x_{a}\right) d x$

Subject to: $\sum_{k \in K_{r s}} f_{k}=q_{r s} \quad \forall r s$

$\sum_{r s} \sum_{k \in K_{r s}} \delta_{a k} f_{k}=x_{a} \quad \forall a \in A$

$f_{k} \geq 0 \quad \forall k \in K_{r s}, r s$

Second:

Minimize $Z_{2}=\sum_{a} \beta_{a} x_{a}^{U E} \quad \forall a \in A$

Subject to:

$\sum_{r s} q_{r s} u_{r s} \geq \sum_{a}\left(t_{a}\left(x_{a}^{U E}\right)+\beta_{a}\right) x_{a}^{U E}$

$u_{r s} \leq \sum_{a} \delta_{a k}\left(t_{a}\left(x_{a}^{U E}\right)+\beta_{a}\right) \quad \forall r s, k \in K_{r s}, r s$

$\beta_{a} \geq 0 \quad \forall_{a}$

The notation in mentioned equations comes as follows:

$Z_{1} \quad$ - function that controls the user equilibrium;

$Z_{2}$ - function that controls the minimum hidden cost;

a - represents a link in the transportation network;

$x_{a} \quad$ - flow in link $a$;

$x_{a}^{U E} \quad$ - flow in link a based on user equilibrium (UE) condition, obtained from stage one;

$t_{a}\left(x_{a}\right)$ - Travel Time in link $a$;

$\beta_{a}-$ toll on link $a$;

$k \quad$ - feasible paths in the network;

$f_{k} \quad$ - flow in path $k$;

rs - Origin-Destination set in the network;

$q_{r s}$ - Demand Matrix between $r s$;

$\delta_{a k}=\left\{\begin{array}{l}1 \text { for each } a \in f_{k} \\ 0\end{array}\right.$

$u_{r s} \quad$ - Travel Time in shortest path between $r s$;

$K_{r s}$ - set of feasible paths between $r$.

This model is solved in two stages, first, Objective 20 is solved, which is the user equilibrium traffic assignment that shows the summation of the flows based on the travel times of each link. By optimizing this function, the model tries to reach optimized user equilibrium condition of traffic flow. Constraint 21 ensures that the demand of each pair of origin-destination is satisfied; in other words, the summation of all flows in all origin-destination pairs is equal to the total demand. Constraint 22 makes the aggregated flow become equal to the optimized system flow in all of the network links. Constraint 23 limits the paths flow to non-negative flows.

In the second stage, objective 24 tries to minimize the total hidden cost by considering the number of tolled links. Constraints 25 and 26 are supplementary ones and cause that each origin-destination pair $r s$, only the routes $k \in K_{r s}$ which cost equal to the shortest route based on the lowest cost $u_{r s}$ have non-zero flow. Constraint 27 shows that the congestion toll 
obtained for each link in the set of the network links, should be positive.

By solving the model and finding the answer based on the objective functions and the defined constraints, the requirements of first-order condition of optimality are practically satisfied. Also, as in all symmetric matrices, like Hessian matrix, if and only if all eigenvalues of the matrix be positive, then the matrix is positive-definite [18]. Thus, the Hessian matrix for controlling the requirements for second-order condition of optimality is checked, and as the function is convex for all variables, so, the hessian matrix of the model is positive-definite and the uniqueness of the answer is controlled.

\subsection{Numerical simulation network}

This paper employed the nine-node network that was used in many research literature [21-23, 25, 2931] as a testbed for providing a better comparison of the MHCCUE model versus the previous ones that had a solution based on UE and SO for this network. The network topology is presented in Figure 1.

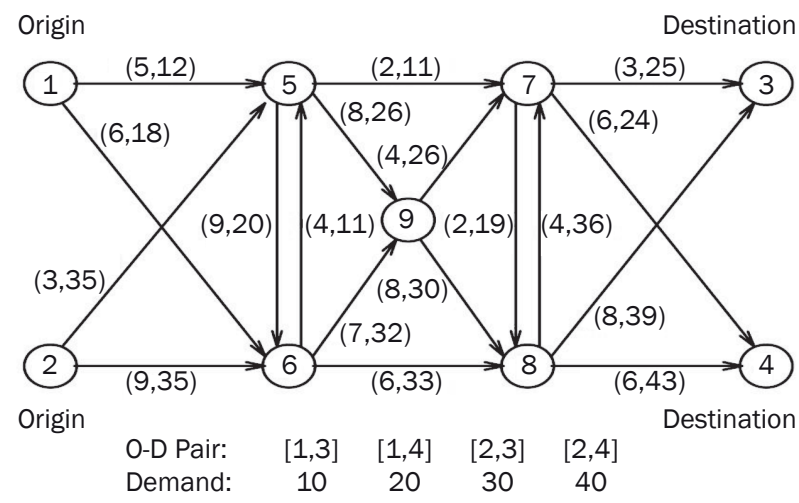

Figure 1 - Nine-Node network: the tuple near link a is (Ta,Ca)

As shown in Figure 1, the network has nine nodes, eighteen links with the same structure of cost function, and four pairs of origin-destination (OD): $(1,3),(1,4)$, $(2,3)$ and $(2,4)$. As shown in the bottom of Figure 1 , the demand of node one to node three equals 10 travels; node one to node four equals 20 travels; node two to node three equals 30 travels, and node two to node four equals 40 travels. The link performance function or its time-travel (TT) is based on the Bureau of Public Roads (BPR) function as follows:

$t_{a}=T_{a}\left[1+0.15\left(\frac{x_{a}}{C_{a}}\right)^{4}\right]$

The parameters $T_{a}$ and $C_{a}$ are constant values, which respectively represent free flow travel time and practical capacity of the links in the network, and their values are shown next to each link. For example, for the link (arc) from node 1 to 5 , the parameters are $\left(T_{a}, C_{a}\right) \equiv(5,12)$; it means that the free flow travel time is 5 and the practical capacity is 12 for the mentioned link.

\subsection{Solution results}

Table 1 shows the results of running the codified model in Maple, that is obtained by solving the minimization hidden cost of congestion based on the user equilibrium (MHCCUE) model. The user equilibrium assignment as the first stage of formulation showed the flow pattern $x_{a}^{U E}$ of the network. This program includes a convex objective function and a linear constraint, so it is solved based on the convex combinations algorithm. This algorithm is mostly appropriate for solving the UE program since the direction finding step can be executed relatively efficiently [18]. The algorithm can be summarized as follows:

Step 0: Initialization. Perform all-or-nothing assignment based on free flow travel times. This yields initial flows. Set counter $n:=1$.

Step 1: Update travel time for each link based on BPR function.

Step 2: Direction finding. Perform all-or-nothing assignment based on the updated travel times and find the auxiliary flows $y_{a}^{n}$.

Step 3: Line search. Finding a coefficient $\left(0 \leq \alpha_{n} \leq 1\right)$ that solves $\min \sum_{a} \int_{0}^{x_{a}^{n}+\alpha\left(y_{a}^{n}-x_{a}^{n}\right)} t_{a}(\omega) d x$

Step 4: Move. Set $x_{a}^{n+1}=x_{a}^{n}+a_{n}\left(y_{a}^{n}-x_{a}^{n}\right)$

Step 5: Convergence test. If a convergence criterion is met, stop (the $x_{a}^{n+1}$ is the set of equilibrium link flows); otherwise, set $n=n+1$ and go to step 1 .

The Table has seven columns, which respectively from left to right include link numbers in which 18 links are numbered based on the network topology. The next column is Arc; it shows the start and end node of the links. Flow $x_{a}^{U E}$ column practically shows the flows obtained from the user equilibrium objective function, which means the flow is assigned based on the user equilibrium to find the minimum travel time by considering this assumption that they know traffic situation in the long term.

The travel time $\left(t_{a}\left(x_{a}^{U E}\right)\right)$ column shows the travel time in UE condition based on its flow $\left(x_{a}^{U E}\right)$. The total travel time $\left(x_{a}^{U E} \cdot t_{a}\left(x_{a}^{U E}\right)\right)$ column is calculated by multiplying the flow by travel time for each link. Toll $\left(\beta_{a}\right)$ column shows the calculation of tolls based on the second-stage objective function $\left(Z_{2}\right)$, and finally the total hidden cost $\left(\beta_{a} \cdot x_{a}^{U E}\right)$ column is considered as the multiplication of the toll $\left(\beta_{a}\right)$ by UE flow $\left(x_{a}^{U E}\right)$.

As shown in Table 1, the model finds the two potential links that could be considered for implementing the TDM policies. In fact, these two links are the critical links in the network which the system should consider for placing control on their demand. In other words, the network could be in an equilibrium condition if the system imposed the tolls on the introduced links 
Table 1 - Results of the MHCCUE model for the Nine-node network

\begin{tabular}{|c|c|c|c|c|c|c||}
\hline $\begin{array}{c}\text { Link } \\
\forall_{a} \in A\end{array}$ & Arc & $\begin{array}{c}\text { Flow } \\
x_{a}^{U E}\end{array}$ & $\begin{array}{c}\text { Travel Time } \\
t_{a}\left(x_{a}^{U E}\right)\end{array}$ & $\begin{array}{c}\text { Total Travel Time } \\
x_{a}^{U E} \cdot t_{a}\left(x_{a}^{U E}\right)\end{array}$ & $\begin{array}{c}\text { Toll } \\
\beta_{a}\end{array}$ & $\begin{array}{c}\text { Total Hidden Cost } \\
\beta_{a} \cdot x_{a}^{U E}\end{array}$ \\
\hline \hline 1 & $1-5$ & 8.160 & 5.160 & 42.106 & 2.194 & 17.903 \\
\hline 2 & $1-6$ & 21.840 & 7.951 & 173.648 & 0.000 & 0.000 \\
\hline 3 & $2-5$ & 47.372 & 4.510 & 213.661 & 0.000 & 0.000 \\
\hline 4 & $2-6$ & 22.628 & 9.236 & 208.985 & 0.000 & 0.000 \\
\hline 5 & $5-6$ & 0.000 & 9.000 & 0.000 & 0.000 & 0.000 \\
\hline 6 & $5-7$ & 27.843 & 14.315 & 398.583 & 0.000 & 0.000 \\
\hline 7 & $5-9$ & 27.689 & 9.543 & 264.244 & 0.000 & 0.000 \\
\hline 8 & $6-5$ & 0.000 & 4.000 & 0.000 & 0.000 & 0.000 \\
\hline 9 & $6-8$ & 44.468 & 8.967 & 398.763 & 7.097 & 315.578 \\
\hline 10 & $6-9$ & 0.000 & 7.000 & 0.000 & 0.000 & 0.000 \\
\hline 11 & $7-3$ & 38.160 & 5.443 & 207.690 & 0.000 & 0.000 \\
\hline 12 & $7-4$ & 17.372 & 6.247 & 108.527 & 0.000 & 0.000 \\
\hline 13 & $7-8$ & 0.000 & 2.000 & 0.000 & 0.000 & 0.000 \\
\hline 14 & $8-3$ & 1.840 & 8.000 & 14.724 & 0.000 & 0.000 \\
\hline 15 & $8-4$ & 42.628 & 6.869 & 292.818 & 0.000 & 0.000 \\
\hline 16 & $8-7$ & 0.000 & 4.000 & 0.000 & 0.000 & 0.000 \\
\hline 17 & $9-7$ & 27.689 & 4.772 & 132.122 & 0.000 & 0.000 \\
\hline 18 & $9-8$ & 0.000 & 8.000 & 0.000 & 0.000 & 0.000 \\
\hline
\end{tabular}

(No. 1 and 9). Also, the traffic managers could distinguish their preferences based on the amount of the hidden cost defined by the model calculation. In this regard, the link with the bigger hidden costs, which in this case is link number 9 , should be considered as the first potential link for TDM policies implementation.

\section{COMPARISON OF RESULTS}

To confirm that using the MHCCUE performs better in finding the best potential link for implementing TDM strategies than the previous models, first, the SO and UE conditions for the nine-node network are presented in Table 2. Second, the final results of the MCSP, MinSys, MinTB, and MinMax are summarized in Table 3. These outputs are compared with MHCCUE; and the results obtained from this comparison are presented in Table 4.

$x_{a}^{U E}$ - traffic flow based on the user equilibrium (UE); $x_{a}^{S O}$ - traffic flow based on the system optimum (SO).

Table 2 shows the sum of total travel time, which is flow multiplied by travel time, for UE is 2455.871 and it is 2254.143 for SO condition. It means by closing the flow from UE to SO, the sum of total travel time will be decreased. But as we are trying to consider the user behaviour before implementing any TDM policies, we use UE condition. Table 3 shows the results of the mentioned models in literature review for the ninenode network. By comparing the result of the previous models with the suggested model, the preference of the proposed model could be clear. Although the previous models had been concentrated on the congestion toll pricing, in this paper, the suggested model tries to investigate the method to find the possible links for implementing the TDM policies in terms of traffic congestion based on benefit priorities and their estimated costs.

To evaluate the model and ensure that more desirable results are obtained in the proposed model, it has been compared with the Table 3 models. Therefore, the mentioned network in this paper was once solved by UE condition and the solution results were compared with each other in case of finding the minimum number of links. In other words, the second part of objective function $\left(Z_{2}\right)$ has been designed based on minimizing the cost of travel in MHCCUE model and other models. One can conclude that if the MHCCUE model is solved without considering SO condition, but just by using the UE condition, it practically means solving the congestion toll pricing problem. But, as the main goal of these problems is finding the best price based on minimum travel time for the whole network, they need to consider the SO condition. In other words, they minimize the number of tolled links but after implementing SO conditions in their first level of optimization, the MHCCUE model predicts the suitable links before implementing any TDM strategy like congestion pricing. Also, it will minimize the total number of selected links based on its formulation.

Table 4 summarizes the model's final results and compares them with MSCP, MinSys, MinMax, and 
S. Afandizadeh Zargari, et al.: Quick Link Selection Method by Using Pricing Strategy Based on User Equilibrium for Implementing...

Table 2 - UE and SO results for Nine-node problem

\begin{tabular}{||c|c|c|c|c|c|c|c||}
\hline \multirow{2}{*}{$\begin{array}{c}\text { Link } \\
\forall_{a} \in A\end{array}$} & \multirow{2}{*}{ Arc } & \multicolumn{3}{|c|}{ UE Condition } & \multicolumn{3}{c||}{ SO Condition } \\
\cline { 3 - 8 } & & $\begin{array}{c}\text { Flow } \\
x_{a}^{U E}\end{array}$ & $\begin{array}{c}\text { Travel Time } \\
t_{a}\left(x_{a}^{U E}\right)\end{array}$ & $\begin{array}{c}\text { Total Travel } \\
\text { Time } \\
x_{a}^{U E} \cdot t_{a}\left(x_{a}^{U E}\right)\end{array}$ & $\begin{array}{c}\text { Flow } \\
x_{a}^{S O}\end{array}$ & $\begin{array}{c}\text { Travel Time } \\
t_{a}\left(x_{a}^{S O}\right)\end{array}$ & $\begin{array}{c}\text { Total Travel } \\
\text { Time } \\
x_{a}^{S O} \cdot t_{a}\left(x_{a}^{S O}\right)\end{array}$ \\
\hline \hline 1 & $1-5$ & 8.160 & 5.160 & 42.106 & 9.411 & 5.284 & 49.728 \\
\hline 2 & $1-6$ & 21.840 & 7.951 & 173.648 & 20.589 & 7.541 & 155.262 \\
\hline 3 & $2-5$ & 47.372 & 4.510 & 213.661 & 38.334 & 3.648 & 139.842 \\
\hline 4 & $2-6$ & 22.628 & 9.236 & 208.985 & 31.666 & 9.905 & 313.652 \\
\hline 5 & $5-6$ & 0.000 & 9.000 & 0.000 & 0.000 & 9.000 & 0.000 \\
\hline 6 & $5-7$ & 27.843 & 14.315 & 398.583 & 21.303 & 6.220 & 132.652 \\
\hline 7 & $5-9$ & 27.689 & 9.543 & 264.244 & 26.442 & 9.284 & 245.487 \\
\hline 8 & $6-5$ & 0.000 & 4.000 & 0.000 & 0.000 & 4.000 & 0.000 \\
\hline 9 & $6-8$ & 44.468 & 8.967 & 398.763 & 39.474 & 7.843 & 309.595 \\
\hline 10 & $6-9$ & 0.000 & 7.000 & 0.000 & 7.000 & 0.000 & 0.000 \\
\hline 11 & $7-3$ & 38.160 & 5.443 & 207.690 & 29.608 & 3.885 & 115.027 \\
\hline 12 & $7-4$ & 17.372 & 6.247 & 108.527 & 20.757 & 6.504 & 135.004 \\
\hline 13 & $7-8$ & 0.000 & 2.000 & 0.000 & 2.000 & 0.000 & 0.000 \\
\hline 14 & $8-3$ & 1.840 & 8.000 & 14.724 & 10.392 & 8.006 & 83.198 \\
\hline 15 & $8-4$ & 42.628 & 6.869 & 292.818 & 39.243 & 6.624 & 259.946 \\
\hline 16 & $8-7$ & 0.000 & 4.000 & 0.000 & 4.000 & 0.000 & 0.000 \\
\hline 17 & $9-7$ & 27.689 & 4.772 & 132.122 & 29.062 & 4.937 & 143.479 \\
\hline 18 & $9-8$ & 0.000 & 8.000 & 0.000 & 10.162 & 8.016 & 81.459 \\
\hline
\end{tabular}

Table 3 - Results of MSCP, MinSys, MinMax, and MinTB models for Nine-node network [21-23, 25].

\begin{tabular}{|c|c|c|c|c|c|c|c|c|c|}
\hline \multirow[b]{2}{*}{$\underset{\forall}{\stackrel{\text { Link }}{\forall} \in A}$} & \multirow[b]{2}{*}{ Arc } & \multicolumn{2}{|c|}{ MSCP } & \multicolumn{2}{|c|}{ MinSys } & \multicolumn{2}{|c|}{ MinMax } & \multicolumn{2}{|c|}{ MinTB } \\
\hline & & $\begin{array}{l}\text { Toll } \\
\beta_{a}\end{array}$ & $\begin{array}{c}\text { Total Hid- } \\
\text { den Cost } \\
\beta_{a} \cdot x_{a}^{\text {so }}\end{array}$ & $\begin{array}{c}\text { Toll } \\
\beta_{a}\end{array}$ & $\begin{array}{l}\text { Total Hid- } \\
\text { den Cost }\end{array}$ & $\begin{array}{c}\text { Toll } \\
\beta_{a}\end{array}$ & $\begin{array}{c}\text { Total Hid- } \\
\text { den Cost } \\
\beta_{a} \cdot x_{a}^{\text {sO }}\end{array}$ & $\begin{array}{c}\text { Toll } \\
\beta_{a}\end{array}$ & $\begin{array}{c}\text { Total Hid- } \\
\text { den Cost } \\
\beta_{a} \cdot x_{a}^{\text {SO }}\end{array}$ \\
\hline 1 & $1-5$ & 1.135 & 10.681 & 0.000 & 0.000 & 0.000 & 0.000 & 0.000 & 0.000 \\
\hline 2 & $1-6$ & 6.162 & 126.869 & 0.000 & 0.000 & 8.000 & 164.712 & 0.000 & 0.000 \\
\hline 3 & $2-5$ & 2.590 & 99.285 & 4.000 & 153.336 & 4.000 & 153.336 & 4.000 & 153.336 \\
\hline 4 & $2-6$ & 3.618 & 114.566 & 0.000 & 0.000 & 0.000 & 0.000 & 0.000 & 0.000 \\
\hline 5 & $5-6$ & 0.000 & 0.000 & 0.000 & 0.000 & 0.000 & 0.000 & 0.000 & 0.000 \\
\hline 6 & $5-7$ & 16.880 & 359.595 & 11.200 & 238.594 & 8.000 & 170.424 & 11.200 & 238.594 \\
\hline 7 & $5-9$ & 5.135 & 135.780 & 0.000 & 0.000 & 0.000 & 0.000 & 0.000 & 0.000 \\
\hline 8 & $6-5$ & 0.000 & 0.000 & 0.000 & 0.000 & 0.000 & 0.000 & 0.000 & 0.000 \\
\hline 9 & $6-8$ & 7.370 & 290.923 & 7.2 & 284.213 & 7.200 & 284.213 & 7.2 & 284.213 \\
\hline 10 & $6-9$ & 0.107 & 1.368 & 0.000 & 0.000 & 0.000 & 0.000 & 0.000 & 0.000 \\
\hline 11 & $7-3$ & 3.541 & 104.841 & 4.000 & 118.432 & 7.200 & 213.178 & 4.000 & 118.432 \\
\hline 12 & $7-4$ & 2.014 & 41.805 & 0.000 & 0.000 & 3.200 & 66.422 & 0.000 & 0.000 \\
\hline 13 & $7-8$ & 0.000 & 0.000 & 0.000 & 0.000 & 1.079 & 0.000 & 0.000 & 0.000 \\
\hline 14 & $8-3$ & 0.024 & 0.249 & 0.000 & 0.000 & 0.000 & 0.000 & 0.000 & 0.000 \\
\hline 15 & $8-4$ & 2.497 & 97.990 & 0.000 & 0.000 & 0.000 & 0.000 & 0.000 & 0.000 \\
\hline 16 & 8-7 & 0.000 & 0 & 0.000 & 0.000 & 0.000 & 0.000 & 0.000 & 0.000 \\
\hline 17 & $9-7$ & 3.746 & 108.866 & 3.200 & 92.998 & 0.000 & 0.000 & 3.200 & 92.998 \\
\hline 18 & $9-8$ & 0.063 & 0.640 & 0.000 & 0.000 & 0.000 & 0.000 & 0.000 & 0.000 \\
\hline
\end{tabular}


Table 4 - Results of the suggested model (MHCCUE) in comparison with other studies

\begin{tabular}{||c|c|c|c|c||}
\hline Solutions & No. of Selected Links & Total Hidden Cost & Total Travel Time & Total Cost \\
\hline \hline MSCP & 14 & $1,493.458$ & $2,253.918$ & $3,747.376$ \\
\hline MinSys & 5 & 887.574 & $2,253.918$ & $3,141.492$ \\
\hline MinMax & 7 & $1,167.572$ & $2,253.918$ & $3,421.490$ \\
\hline MinTB & 5 & 887.574 & $2,253.918$ & $3,141.492$ \\
\hline MHCCUE & 2 & 333.482 & $2,455.870$ & $2,789.351$ \\
\hline
\end{tabular}

MinTB, since they have been used in many pricing studies with the same testbed [21-23, 25]. It shows that the total cost in MHCCUE model is 2,789.351 which covers the total travel time which is $2,455.870$ plus total hidden cost which is 333.482 . As shown, not only the number of links that need to be modified by TDM policies is decreased in this model in comparison with the others, but also the total cost of the MHCCUE model has been reduced when compared with the mentioned models.

As shown in Table 4, the number of tolled links decreased from 14 to 2 in MSCP model to MHCCUE model. Since, both traditional or the more modern toll sensing stations, are costly, decreasing the number of tolled links became the main concerns of traffic managers. Moreover, by using the new technology like Global Positioning System (GPS) that allows accurate determination of location, rate, direction, and time of trips, operators could track the user routes. In this regard, it would be interesting for traffic managers to reduce the number of tolled links as much as possible and return back to optimum condition.

Also, Figure 2 shows in the case of applying MHCCUE with the consideration of user equilibrium conditions for the nine-node network, the total hidden cost and the total cost decrease by about $77 \%$ and $25 \%$, respectively. However, the total travel time increases about $9 \%$ for the nine-node network.

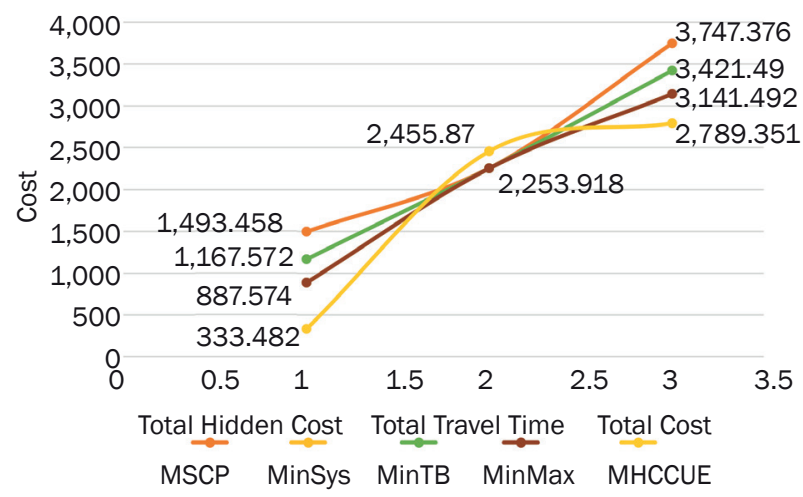

Figure 2 - Total cost of suggested model compared with Previous Studies

On the other hand, Table 4 and Figure 2 also show that by applying the MHCCUE model, not only is the number of tolled links reduced but also at the same level of total travel time due to equilibrium condition, congestion and obstruction state will not occur in the network.

\section{CONCLUSIONS}

This paper presents a new model for choosing the potential location for implementing the TDM policies in urban transportation networks based on the link priority condition. The concept of minimizing the hidden cost of congestion based on user equilibrium (MHCCUE) model is presented in this regard. As the model is a well-defined optimization problem in its first step, which is traffic assignment based on users equilibrium, urban planners and transportation engineers can potentially use this model as a quick estimation tool for deciding on which location to implement the TDM strategies in the urban transportation network, since it just takes traffic assignment data as input to predict the best locations.

Although the main goal of this research is not to calculate the congestion toll pricing, the result shows improvements in this area by considering that the calculation is based on the user equilibrium (UE) rather than system optimum (SO). This assumption helps us to select the possible links based on user behaviour. Thus, the hidden cost of congestion becomes more valuable and helps the planner to consider it as a parameter for choosing the potential links to implement the TDM among all of the network links for managing the demand to avoid urban congestion.

Also, as shown in Figure 2, the model reduces the number of links in comparison with the previous studies. It shows that only by implementing the potential TDM policies in two selected links, the network can be close to equilibrium conditions.

Moreover, for better comparison between MHCCUE and previous models, the results of the aforementioned model are shown in Table 3 for the nine-node network. Of course, for better presentation of the results, the amount of total hidden cost, total travel time, and finally total cost are shown in Figure 2. In this figure, the total cost is the summation of the total hidden cost and the total travel time in the network. As shown in Figure. 2, the proposed model has a minimum total cost as compared with the others. 
شهرياز افندى زاده زرگَرى، دانشيار، كَروه حمل و نقل، دانشكده

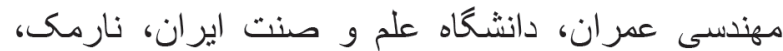

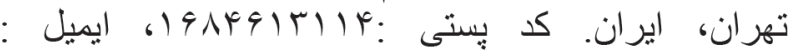
zargari@iust.ac.ir

حمبل مبرزاحسين،(نويسنده مسئول)؛ دانشجوى دكثرى،

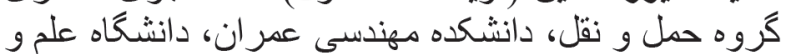

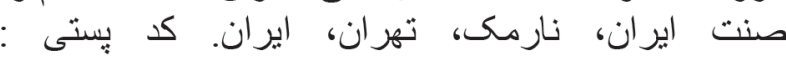

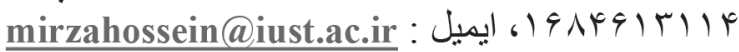

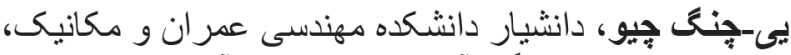

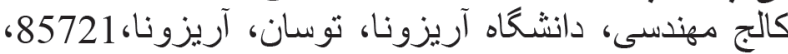

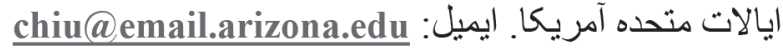

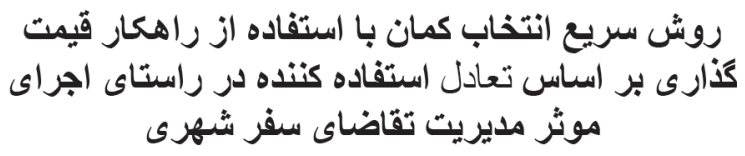

اين مقاله يك مدل بهينه سازى دو مرحله اى را بـه عنو ان

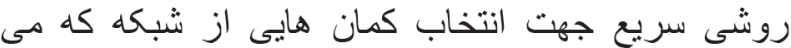
توانتد كانديد مناسبى براي اجر ایى سياست هائ مديريت

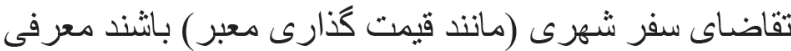

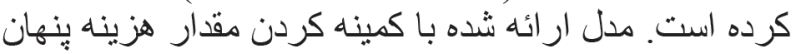

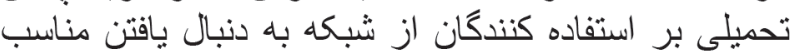

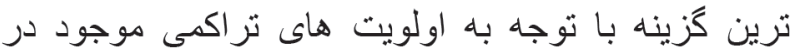

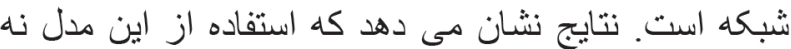

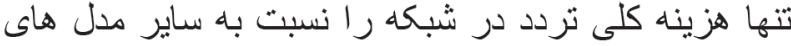

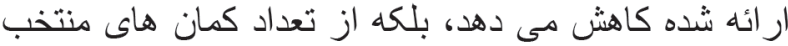

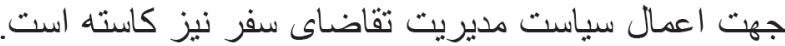

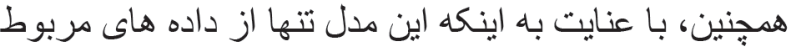
به تخصيص ثر افيك در راستاى انتخاب كمان مناسب بـ بهره

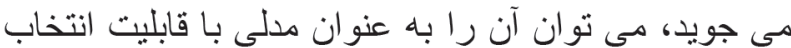

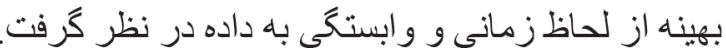

\section{REFERENCES}

[1] Murchland JD. Braess's paradox of traffic flow. Transportation Research. 1970;4(4):391-4.

[2] Afandizadeh SH, Dehghani N, Mirzahossein H, Hamedi M. Improving SCATS operation during congestion periods using internal/external traffic metering strategy. Promet - Traffic \& Transportation. 2016;28(1):41-47.

[3] Jraiw K. Transport demand management - Impacts on congestion alleviation and road safety enhancement in urban areas. Journal of Local and Global Heath Science. 2015(2):83.

[4] Luten K, Binning K, Driver D, Hall T, Schreffler E. Mitigating traffic congestion - the role of demand-side strategies. US Department of Transportation, Federal Highway Administration; 2004.

[5] Ungemah D, Dusza C. Transportation demand management benchmark. Transportation Research Record: Journal of the Transportation Research Board. 2009;2118(1):55-66.
[6] Triantis K, Sarangi S, Teodorović D, Razzolini L. Traffic congestion mitigation: combining engineering and economic perspectives. Transportation Planning and Technology. 2011;34(7):637-45.

[7] Shaw J. The implementation and effectiveness of transport demand management measures: an international perspective. Journal of Transport Geography. 2010;18(6):762.

[8] Broaddus A, Litman T, Menon G. Transportation demand management: Training document. Eschborn, Germany: Federal Ministry for Economic Cooperation and Development; 2009.

[9] Saleh W, Sammer G. Travel demand management and road user pricing: Success, failure and feasibility. Ashgate Publishing, Ltd.; 2012.

[10] De Lara M, de Palma A, Kilani M, Piperno S. Congestion pricing and long term urban form: Application to Paris region. Regional Science and Urban Economics. 2013;43(2):282-95.

[11] Yildirim MB. Congestion toll pricing models and methods for variable demand networks. University of Florida; 2001.

[12] Tezcan HO. Evaluating road pricing with an engineering perspective: aggregate and disaggregate analysis. Canadian Journal of Civil Engineering. 2009;36(6):1028-36.

[13] Small KA, Verhoef ET. The economics of urban transportation: Routledge; 2007.

[14] Pigou AC. The economics of welfare. 4th ed. London: Macmillan; 1920.

[15] Knight FH. Some fallacies in the interpretation of social cost. Quarterly Journal of Economics. 1924;38(4): 582-606.

[16] Wardrop JG. Some theoretical aspects of road traffic research. Proceedings of the Institution of Civil Engineers. 1952;1(3):325-362.

[17] Beckmann M, McGuire C, Winsten CB. Studies in the Economics of Transportation; New Haven: Yale University Press; 1956.

[18] Sheffi Y. Urban transportation networks: equilibrium analysis with mathematical programming methods. Englewood Cliffs, New Jersey: Prentice-Hall, Inc.; 1985.

[19] De Palma A, Lindsey R. Traffic congestion pricing methods and technologies. HAL archive; 2009. Available from: https://hal.archives-ouvertes.fr/hal-00414526/ document

[20] Yang $\mathrm{H}$, Huang $\mathrm{H}-\mathrm{J}$. Principle of marginal-cost pricing: how does it work in a general road network? Transportation Research Part A: Policy and Practice. 1998;32(1):45-54.

[21] Bergendorff P, Hearn DW, Ramana MV. Congestion toll pricing of traffic networks. Springer; 1997.

[22] Hearn DW, Ramana MV. Solving congestion toll pricing models. Springer; 1998.

[23] Hearn DW, Yildirim MB. A toll pricing framework for traffic assignment problems with elastic demand. Springer; 2002.

[24] Patriksson M, Rockafellar RT. A mathematical model and descent algorithm for bilevel traffic management. Transportation Science. 2002;36(3):271-91.

[25] Yildirim MB, Hearn DW. A first best toll pricing framework for variable demand traffic assignment 
problems. Transportation Research Part B: Methodological. 2005;39(8):659-78.

[26] Florian M, Hearn D. Network equilibrium models and algorithms. Handbooks in Operations Research and Management Science. 1995;8:485-550.

[27] Thorpe N, Hills P, Jaensirisak S. Public attitudes to TDM measures: a comparative study. Transport Policy. 2000;7(4):243-57.

[28] Goodwin P, Lyons G. Public attitudes to transport: interpreting the evidence. Transportation planning and technology. 2010;33(1):3-17.
[29] Gendreau M, Lucotte M, editors. Transportation and network analysis: Current trends - Miscellanea in honor of Michael Florian. Springer Science \& Business Media; 2013.

[30] Pardalos P, Hearn D, Hager WW. Network optimization. Springer; 1997.

[31] Hearn D, Ribera J. Bounded flow equilibrium problems by penalty methods. Proceedings of IEEE International Conference on Circuits and Computers; 1980. 